\title{
Second-order constitutive relations for transversely isotropic piezoelectric porous materials
}

\author{
R. C. Batra \\ Department of Engineering Science and Mechanics, Virginia Polytechnic Institute and State University, \\ Blacksburg, Virginia 24061-0219
}

J. S. Yang

Department of Mechanical Engineering, Aeronautical Engineering and Mechanics, Rensselaer Polytechnic Institute, Troy, New York 12180

(Received 10 August 1994; revised 28 October 1994; accepted 28 December 1994)

\begin{abstract}
Based on the theory of invariants, polynomial constitutive relations for transversely isotropic piezoelectric porous materials are derived from the polynomial integrity bases for an energy density function depending on a symmetric second-order tensor and two vectors. They are assumed to be smooth functions of their arguments, are expanded about the values their arguments take in the reference configuration and all terms up to the quadratic terms in the gradients of the mechanical displacement, the electric potential, and the gradients of the volume fraction are kept. The second-order constitutive relations so obtained are then specialized to the case of infinitesimal deformations and weak electric fields, and also to the case of infinitesimal deformations and strong electric fields.
\end{abstract}

PACS numbers: 43.20.Jr, 43.25.Dc, 43.38.Fx

\section{INTRODUCTION}

The effect of nonlinearity in the constitutive relations of piezoelectric ceramics has been of recent interest because of their use in smart structures. Nelson ${ }^{1}$ has given, for all crystal classes, representations of quadratic piezoelectric constitutive relations generated by an energy density function of a symmetric second-order tensor and a vector.

Many piezoelectric materials are porous. ${ }^{2-4}$ Here, based on the theory of invariants, nonlinear form invariant polynomial constitutive relations for transversely isotropic piezoelectric porous materials are derived. They are then reduced to second-order and linear constitutive relations, and constitutive relations for small deformations and strong electric fields.

\section{EQUATIONS FOR A NONLINEAR PIEZOELECTRIC POROUS MATERIAL}

Let the coordinates of a material particle with respect to a rectangular Cartesian coordinate system be $X_{K}$ in the reference configuration, its spatial coordinates in the current configuration be $x_{k}$, then the balance laws for a nonlinear piezoelectric porous material are $\mathrm{a}^{5-6}$

$$
\begin{aligned}
& \rho_{0}=\rho \mathscr{J}, \quad \mathscr{J}=\operatorname{det}\left(x_{k, K}\right), \\
& {\left[T_{K L} x_{k, L}+\mathscr{J} X_{K, l} \epsilon_{0}\left(E_{k} E_{l}-\frac{1}{2} E_{m} E_{m} \delta_{k l}\right)\right]_{, K}+\rho_{0} f_{k}} \\
& \quad=\rho_{0} \delta_{k K} \ddot{U}_{K}, \\
& \left(\Pi_{K}+\mathscr{J} X_{K, k} \epsilon_{0} E_{k}\right)_{, K}=0, \\
& h_{K, K}+\rho_{0}(l+g)=\rho_{0}(k \dot{\psi}),
\end{aligned}
$$

where $\rho$ is the mass density, $\rho_{0}$ is the mass density of the porous material in the reference configuration, $T_{K L}$ is the second Piola-Kirchhoff stress tensor, $U_{K}$ is the mechanical displacement vector, $\delta_{k K}$ is the shifter, $\Pi_{K}$ is the material electric polarization, $E_{k}=-\phi_{, k}$ is the electric field, $\phi$ is electric potential, $\epsilon_{0}$ is the permittivity of the free space, $\delta_{k l}$ is the Kronecker delta, $h_{K}$ is the equilibrated stress, $g$ is the intrinsic equilibrated body force, $l$ is the extrinsic equilibrated body force, $k$ is the equilibrated inertia, and $\psi$ is the volume fraction of voids or the porosity of the material. Throughout this paper, a repeated index implies summation over the range of the index, and a comma followed by $K$ (i) implies partial differentiation with respect to $X_{K}\left(x_{i}\right)$. A dot above a quantity signifies its material time derivative. Balance laws (1) are accompanied by constitutive relations

$$
T_{K L}=\frac{\partial \Sigma}{\partial E_{K L}}, \quad \Pi_{K}=-\frac{\partial \Sigma}{\partial W_{K}}, \quad h_{K}=\frac{\partial \Sigma}{\partial V_{K}},
$$

where $\Sigma\left(E_{K L}, W_{K}, V_{K}\right)$ is an energy density function that also depends on $\psi$, that dependence is not written explicitly. In Eq. (2) $E_{K L}$ is the Green-Lagrange strain tensor, $W_{K}$ is the electric field in material form, and $V_{K}$ is the material gradient of $\psi$.

$E_{K L}=\frac{1}{2}\left(U_{K, L}+U_{L, K}+U_{M, K} U_{M, L}\right)$, $W_{K}=x_{k, K} E_{k}=-x_{k, K} \phi_{, k}=-\phi_{, K} ; \quad V_{K}=x_{k, K} \psi_{, k}=\psi_{, K}$.

\section{FORM INVARIANT POLYNOMIAL CONSTITUTIVE RELATIONS}

Let the material be invariant under rotations about a unit vector $\mathbf{a}$ and reflections about planes containing $\mathbf{a}$. Then any scalar polynomial function of a symmetric tensor $\mathbf{E}$ and two vectors $\mathbf{W}$ and $\mathbf{V}$ must be a polynomial function of the following invariants called the polynomial integrity bases: ${ }^{7}$

$$
\begin{aligned}
& I_{1}=\mathbf{a} \cdot \mathbf{E} \cdot \mathbf{a}, \quad I_{2}=\operatorname{tr} \mathbf{E}, \quad I_{3}=\mathbf{a} \cdot \mathbf{W}, \quad I_{4}=\mathbf{a} \cdot \mathbf{V}, \\
& I I_{1}=\mathbf{a} \cdot \mathbf{E}^{2} \cdot \mathbf{a}, \quad I I_{2}=\operatorname{tr} \mathbf{E}^{2}, \quad I I_{3}=\mathbf{W} \cdot \mathbf{W}, \\
& I I_{4}=\mathbf{a} \cdot \mathbf{E} \cdot \mathbf{W}+\mathbf{W} \cdot \mathbf{E} \cdot \mathbf{a}, \quad I I_{5}=\mathbf{V} \cdot \mathbf{V}, \\
& I I_{6}=\mathbf{a} \cdot \mathbf{E} \cdot \mathbf{V}+\mathbf{V} \cdot \mathbf{E} \cdot \mathbf{a}, \quad I I_{7}=\mathbf{W} \cdot \mathbf{V},
\end{aligned}
$$


$I I I_{1}=\operatorname{tr} \mathbf{E}^{3}, \quad I I I_{2}=\mathbf{W} \cdot \mathbf{E} \cdot \mathbf{W}$,

$I_{3}=\mathbf{a} \cdot \mathbf{E}^{2} \cdot \mathrm{W}+\mathrm{W} \cdot \mathbf{E}^{2} \cdot \mathbf{a}, \quad I I_{4}=\mathrm{V} \cdot \mathbf{E} \cdot \mathrm{V}$,

$I I I_{5}=\mathbf{a} \cdot \mathbf{E}^{2} \cdot \mathrm{V}+\mathrm{V} \cdot \mathbf{E}^{2} \cdot \mathbf{a}, \quad I I I_{6}=\mathbf{W} \cdot \mathbf{E} \cdot \mathrm{V}+\mathbf{V} \cdot \mathbf{E} \cdot \mathbf{W}$,

where $\mathbf{a} \cdot \mathbf{b}$ indicates the inner product between vectors $\mathbf{a}$ and $\mathbf{b}$, and $\operatorname{tr} \mathbf{E}$ equals the sum of the diagonal terms of $\mathbf{E}$. With (4), any scalar form invariant polynomial function of the symmetric tensor $\mathbf{E}$ and vectors $\mathbf{W}$ and $\mathbf{V}$ can be written as

$$
\Sigma=\Sigma\left(I_{1}, \ldots, I_{4}, I I_{1}, \ldots, I I_{7}, I I I_{1}, \ldots, I I I_{6}\right),
$$

where $\Sigma$ is a general polynomial function of its arguments. From (2) and (5), we obtain the following general form for the polynomial constitutive relations for a nonlinear transversely isotropic material:

$$
\begin{aligned}
\mathbf{T}= & \frac{\partial \Sigma}{\partial I_{1}} \mathbf{a} \otimes \mathbf{a}+\frac{\partial \Sigma}{\partial I_{2}} \mathbf{1}+\frac{\partial \Sigma}{\partial I I_{1}}(\mathbf{a} \otimes \mathbf{E} \cdot \mathbf{a}+\mathbf{a} \cdot \mathbf{E} \otimes \mathbf{a})+2 \frac{\partial \Sigma}{\partial I_{2}} \mathbf{E}+\frac{\partial \Sigma}{\partial I_{4}}(\mathbf{a} \otimes \mathbf{W}+\mathbf{W} \otimes \mathbf{a})+\frac{\partial \Sigma}{\partial I_{6}}(\mathbf{a} \otimes \mathbf{V}+\mathbf{V} \otimes \mathbf{a})+3 \frac{\partial \Sigma}{\partial I I I_{1}} \mathbf{E} 2 \\
& +\frac{\partial \Sigma}{\partial I I I_{2}} \mathbf{W} \otimes \mathbf{W}+\frac{\partial \Sigma}{\partial I I_{4}} \mathbf{V} \otimes \mathbf{V}+\frac{\partial \Sigma}{\partial I I_{6}}(\mathbf{W} \otimes \mathbf{V}+\mathbf{V} \otimes \mathbf{W})+\frac{\partial \Sigma}{\partial I I_{3}}(\mathbf{a} \otimes \mathbf{E} \cdot \mathbf{W}+\mathbf{W} \cdot \mathbf{E} \otimes \mathbf{a}+\mathbf{W} \otimes \mathbf{E} \cdot \mathbf{a}+\mathbf{a} \cdot \mathbf{E} \otimes \mathbf{W}) \\
& +\frac{\partial \Sigma}{\partial I I I_{5}}(\mathbf{a} \otimes \mathbf{E} \cdot \mathbf{V}+\mathbf{V} \cdot \mathbf{E} \otimes \mathbf{a}+\mathbf{V} \otimes \mathbf{E} \cdot \mathbf{a}+\mathbf{a} \cdot \mathbf{E} \otimes \mathbf{V}), \\
-\mathbf{I} & =\frac{\partial \Sigma}{\partial I_{3}} \mathbf{a}+2 \frac{\partial \Sigma}{\partial I I_{4}} \mathbf{E} \cdot \mathbf{a}+2 \frac{\partial \Sigma}{\partial I_{3}} \mathbf{W}+\frac{\partial \Sigma}{\partial I I_{7}} \mathbf{V}+2 \frac{\partial \Sigma}{\partial I I_{3}} \mathbf{E}^{2} \cdot \mathbf{a}+2 \frac{\partial \Sigma}{\partial I I I_{2}} \mathbf{E} \cdot \mathbf{W}+2 \frac{\partial \Sigma}{\partial I I I_{6}} \mathbf{E} \cdot \mathbf{V}, \\
\mathbf{h}= & \frac{\partial \Sigma}{\partial I_{4}} \mathbf{a}+2 \frac{\partial \Sigma}{\partial I I_{6}} \mathbf{E} \cdot \mathbf{a}+\frac{\partial \Sigma}{\partial I I_{7}} \mathbf{W}+2 \frac{\partial \Sigma}{\partial I I_{5}} \mathbf{V}+2 \frac{\partial \Sigma}{\partial I I I_{5}} \mathbf{E}^{2} \cdot \mathbf{a}+2 \frac{\partial \Sigma}{\partial I I I_{6}} \mathbf{E} \cdot \mathbf{W}+2 \frac{\partial \Sigma}{\partial I I I_{4}} \mathbf{E} \cdot \mathbf{V},
\end{aligned}
$$

where $\mathbf{1}$ is the identity tensor and $\mathbf{u} \otimes \mathbf{v}$ denotes tensor product between tensors $\mathbf{u}$ and $\mathbf{v}$. In order to derive a second-order theory, we assume that $\Sigma$ is a smooth function of its arguments and write its Taylor series expansion about the values the arguments take in the reference configuration and only keep all terms up to degree three in $\mathbf{E}, \mathbf{W}$, and $\mathbf{V}$ :

$$
\begin{aligned}
\Sigma= & \alpha_{1} I_{1}+\alpha_{2} I_{2}+\beta I_{3}+\gamma I_{4}+c_{1} I_{1}^{2}+c_{2} I_{2}^{2}+c_{3} I_{1} I_{2}+c_{4} I I_{1}+c_{5} I I_{2}+\epsilon_{1} I_{3}^{2}+\epsilon_{2} I I_{3}+\kappa_{1} I_{4}^{2}+\kappa_{2} I I_{5}+e_{1} I_{1} I_{3}+e_{2} I_{2} I_{3}+e_{3} I I_{4} \\
& +f_{1} I_{1} I_{4}+f_{2} I_{2} I_{4}+f_{3} I I_{6}+g_{1} I_{3} I_{4}+g_{2} I I_{7}+\lambda_{1} I_{1}^{3}+\lambda_{2} I_{2}^{3}+\lambda_{3} I_{1}^{2} I_{2}+\lambda_{4} I_{2}^{2} I_{1}+\lambda_{5} I I_{1} I_{1}+\lambda_{6} I I_{1} I_{2}+\lambda_{7} I I_{2} I_{1}+\lambda_{8} I I_{2} I_{2} \\
& +\lambda_{9} I I_{1}+\mu_{1} I_{3}^{3}+\mu_{2} I I_{3} I_{3}+\xi_{1} I_{4}^{3}+\xi_{2} I_{5} I_{4}+\nu_{1} I_{1}^{2} I_{3}+\nu_{2} I_{3}^{2} I_{1}+\nu_{3} I_{2}^{2} I_{3}+\nu_{4} I_{3}^{2} I_{2}+\nu_{5} I I_{1} I_{3}+\nu_{6} I_{2} I_{3}+\nu_{7} I I_{3} I_{1}+\nu_{8} I I_{3} I_{2} \\
& +\nu_{9} I I_{4} I_{1}+\nu_{10} I_{4} I_{2}+\nu_{11} I_{4} I_{3}+\nu_{12} I I I_{2}+\nu_{13} I I_{3}+\nu_{14} I_{1} I_{2} I_{3}+\eta_{1} I_{1}^{2} I_{4}+\eta_{2} I_{4}^{2} I_{1}+\eta_{3} I_{2}^{2} I_{4}+\eta_{4} I_{4}^{2} I_{2}+\eta_{5} I I_{1} I_{4}+\eta_{6} I I_{2} I_{4} \\
& +\eta_{7} I I_{5} I_{1}+\eta_{8} I I_{5} I_{2}+\eta_{9} I I_{6} I_{1}+\eta_{10} I I_{6} I_{2}+\eta_{11} I_{6} I_{4}+\eta_{12} I I I_{4}+\eta_{13} I I I_{5}+\eta_{14} I_{1} I_{2} I_{4}+\zeta_{1} I_{3}^{2} I_{4}+\zeta_{2} I_{4}^{2} I_{3}+\zeta_{3} I I_{3} I_{4} \\
& +\zeta_{4} I I_{5} I_{3}+\zeta_{5} I I_{7} I_{3}+\zeta_{6} I I_{7} I_{4}+\delta_{1} I_{1} I_{3} I_{4}+\delta_{2} I_{2} I_{3} I_{4}+\delta_{3} I I_{4} I_{4}+\delta_{4} I I_{6} I_{3}+\delta_{5} I I_{7} I_{1}+\delta_{6} I I_{7} I_{2}+\delta_{7} I I I_{6},
\end{aligned}
$$

where $\alpha_{1}, \alpha_{2}, \beta$, and $\gamma$ will be shown to represent initial fields. Here, $c_{1}-c_{5}, \epsilon_{1}, \epsilon_{2}, \kappa_{1}, \kappa_{2}, e_{1}-e_{3}, f_{1}-f_{3}, g_{1}$, and $g_{2}$ are 17 constants for the quadratic terms in $\Sigma$ or for the linear constitutive relations; $\lambda_{1}-\lambda_{9}$ represent cubic terms containing $\mathbf{E}$ alone, $\mu_{1}$ and $\mu_{2}$ cubic terms containing $\mathbf{W}$ alone, $\xi_{1}$ and $\xi_{2}$ cubic terms containing $\mathbf{V}$ alone, $\nu_{1}-\nu_{14}$ cubic terms containing $\mathbf{E}$ and $\mathbf{W}, \eta_{1}-\eta_{14}$ cubic terms containing $\mathbf{E}$ and $\mathbf{V}, \zeta_{1}-\zeta_{6}$ cubic terms containing $\mathbf{W}$ and $\mathbf{V}$, and $\delta_{1}-\delta_{7}$ cubic terms containing $\mathbf{E}, \mathbf{W}$, and $\mathbf{V}$. There are 54 constants in all for cubic terms in $\Sigma$ or the quadratic constitutive relations. Substitution of (9) into (6)-(8) gives

$$
\begin{aligned}
\mathbf{T}= & \left(\alpha_{1}+2 c_{1} I_{1}+c_{3} I_{2}+e_{1} I_{3}+f_{1} I_{4}+3 \lambda_{1} I_{1}^{2}+2 \lambda_{3} I_{1} I_{2}+\lambda_{4} I_{2}^{2}+\lambda_{5} I_{1}+\lambda_{7} I_{2}+2 \nu_{1} I_{1} I_{3}+\nu_{2} I_{3}^{2}+\nu_{7} I_{3}+\nu_{9} I I_{4}+\nu_{14} I_{2} I_{3}\right. \\
& \left.+2 \eta_{1} I_{1} I_{4}+\eta_{2} I_{4}^{2}+\eta_{7} I_{5}+\eta_{9} I_{6}+\eta_{14} I_{2} I_{4}+\delta_{1} I_{3} I_{4}+\delta_{5} I_{7}\right) \mathbf{a} \otimes \mathbf{a}+\left(\alpha_{2}+2 c_{2} I_{2}+c_{3} I_{1}+e_{2} I_{3}+f_{2} I_{4}+3 \lambda_{2} I_{2}^{2}+\lambda_{3} I_{1}^{2}\right. \\
& +2 \lambda_{4} I_{1} I_{2}+\lambda_{6} I_{1}+\lambda_{8} I_{2}+2 \nu_{3} I_{2} I_{3}+\nu_{4} I_{3}^{2}+\nu_{8} I_{3}+\nu_{10} I_{4}+\nu_{14} I_{1} I_{3}+2 \eta_{3} I_{2} I_{4}+\eta_{4} I_{4}^{2}+\eta_{8} I_{5}+\eta_{10} I I_{6}+\eta_{14} I_{1} I_{4}+\delta_{2} I_{3} I_{4} \\
& \left.+\delta_{6} I I_{7}\right) \mathbf{1}+\left(c_{4}+\lambda_{5} I_{1}+\lambda_{6} I_{2}+\nu_{5} I_{3}+\eta_{5} I_{4}\right)(\mathbf{a} \otimes \mathbf{E} \cdot \mathbf{a}+\mathbf{a} \cdot \mathbf{E} \otimes \mathbf{a})+2\left(c_{5}+\lambda_{7} I_{1}+\lambda_{8} I_{2}+\nu_{6} I_{3}+\eta_{6} I_{4}\right) \mathbf{E}+\left(e_{3}+\nu_{9} I_{1}+\nu_{10} I_{2}\right. \\
& \left.+\nu_{11} I_{3}+\delta_{3} I_{4}\right)(\mathbf{a} \otimes \mathbf{W}+\mathbf{W} \otimes \mathbf{a})+\left(f_{3}+\eta_{9} I_{1}+\eta_{10} I_{2}+\eta_{11} I_{4}+\delta_{4} I_{3}\right)(\mathbf{a} \otimes \mathbf{V}+\mathbf{V} \otimes \mathbf{a})+3 \lambda_{9} \mathbf{E}^{2}+\nu_{12} \mathbf{W} \otimes \mathbf{W}+\eta_{12} \mathbf{V} \otimes \mathbf{V}+\nu_{13}(\mathbf{a} \\
& \otimes \mathbf{E} \cdot \mathbf{W}+\mathbf{W} \cdot \mathbf{E} \otimes \mathbf{a}+\mathbf{W} \otimes \mathbf{E} \cdot \mathbf{a}+\mathbf{a} \cdot \mathbf{E} \otimes \mathbf{W})+\eta_{13}(\mathbf{a} \otimes \mathbf{E} \cdot \mathbf{V}+\mathbf{V} \cdot \mathbf{E} \otimes \mathbf{a}+\mathbf{V} \otimes \mathbf{E} \cdot \mathbf{a}+\mathbf{a} \cdot \mathbf{E} \otimes \mathbf{V})+\delta_{7}(\mathbf{W} \otimes \mathbf{V}+\mathbf{V} \otimes \mathbf{W}),
\end{aligned}
$$

$$
\begin{aligned}
-\Pi= & \left(\beta+2 \epsilon_{1} I_{3}+e_{1} I_{1}+e_{2} I_{2}+g_{1} I_{4}+3 \mu_{1} I_{3}^{2}+\mu_{2} I I_{3}+\nu_{1} I_{1}^{2}+2 \nu_{2} I_{3} I_{1}+\nu_{3} I_{2}^{2}+2 \nu_{4} I_{3} I_{2}+\nu_{5} I I_{1}+\nu_{6} I I_{2}+\nu_{11} I_{4}+\nu_{14} I_{1} I_{2}\right. \\
& \left.+2 \zeta_{1} I_{3} I_{4}+\zeta_{2} I_{4}^{2}+\zeta_{4} I_{5}+\zeta_{5} I_{7}+\delta_{1} I_{1} I_{4}+\delta_{2} I_{2} I_{4}+\delta_{4} I I_{6}\right) \mathbf{a}+2\left(e_{3}+\nu_{9} I_{1}+\nu_{10} I_{2}+\nu_{11} I_{3}+\delta_{3} I_{4}\right) \mathbf{E} \cdot \mathbf{a} \\
& +2\left(\epsilon_{2}+\mu_{2} I_{3}+\nu_{7} I_{1}+\nu_{8} I_{2}+\zeta_{3} I_{4}\right) \mathbf{W}+\left(g_{2}+\zeta_{5} I_{3}+\zeta_{6} I_{4}+\delta_{5} I_{1}+\delta_{6} I_{2}\right) \mathbf{V}+2 \nu_{13} \mathbf{E}^{2} \cdot \mathbf{a}+2 \nu_{12} \mathbf{E} \cdot \mathbf{W}+2 \delta_{7} \mathbf{E} \cdot \mathbf{V}
\end{aligned}
$$




$$
\begin{aligned}
\mathbf{h}= & \left(\gamma+2 \kappa_{1} I_{4}+f_{1} I_{1}+f_{2} I_{2}+g_{1} I_{3}+3 \xi_{1} I_{4}^{2}+\xi_{2} I I_{5}+\eta_{1} I_{1}^{2}+2 \eta_{2} I_{4} I_{1}+\eta_{3} I_{2}^{2}+2 \eta_{4} I_{4} I_{2}+\eta_{5} I I_{1}+\eta_{6} I I_{2}+\eta_{11} I_{6}+\eta_{14} I_{1} I_{2}\right. \\
& \left.+2 \zeta_{2} I_{3} I_{4}+\zeta_{1} I_{3}^{2}+\zeta_{3} I_{3}+\zeta_{6} I I_{7}+\delta_{1} I_{1} I_{3}+\delta_{2} I_{2} I_{3}+\delta_{3} I_{4}\right) \mathbf{a}+2\left(f_{3}+\eta_{9} I_{1}+\eta_{10} I_{2}+\eta_{11} I_{4}+\delta_{4} I_{3}\right) \mathbf{E} \cdot \mathbf{a} \\
& +\left(g_{2}+\zeta_{5} I_{3}+\zeta_{6} I_{4}+\delta_{5} I_{1}+\delta_{6} I_{2}\right) \mathbf{W}+2\left(\kappa_{2}+\xi_{2} I_{4}+\eta_{7} I_{1}+\eta_{8} I_{2}+\zeta_{4} I_{3}\right) \mathrm{V}+2 \eta_{13} \mathbf{E}^{2} \cdot \mathbf{a}+2 \delta_{7} \mathbf{E} \cdot \mathbf{W}+2 \eta_{12} \mathbf{E} \cdot \mathbf{V}
\end{aligned}
$$

Equations (10)-(12) are polynomial representations of $\mathbf{T}, \boldsymbol{\Pi}$, and $\mathbf{h}$ of degree two in components of $\mathbf{E}, \mathbf{W}$, and $\mathbf{V}$. It can be seen that terms in (10) involving $\alpha_{1}$ and $\alpha_{2}$ do not depend upon $\mathbf{E}, \mathbf{W}$, or $\mathbf{V}$ and hence represent the initial stress. Terms involving $\beta$ in (11) and $\gamma$ in (12) are similar. The derivations in this section are for the constitutive relations generated by an energy density function of a symmetric tensor and two vectors in general. When applied to the case of a piezoelectric porous material, all of the material parameters should be considered as functions of $\psi$, the porosity of the material.

Passman and Batra ${ }^{6}$ assumed that $\mathbf{T}, \Pi$, and $\mathbf{h}$ also depend upon $\dot{\psi}$, the rate of change of the porosity. If we adopt this assumption, then the material parameters will also depend upon $\psi$. Henceforth, we disregard the dependence of $\mathbf{T}$, $\Pi$, and $\mathbf{h}$ upon $\dot{\psi}$.

\section{SECOND-ORDER CONSTITUTIVE RELATIONS}

By second-order constitutive relations we mean relations that contain all quadratic terms of the mechanical displacement gradient, electric potential gradient, and the gradient of the volume fraction of voids. Equations (10)-(12) contain some higher-order terms in this sense. To get second-order constitutive relations, we make the following decompositions:

$$
\mathbf{E}=\mathbf{E}^{(1)}+\mathbf{E}^{(2)}, \quad E_{K L}^{(1)}=\frac{1}{2}\left(U_{K, L}+U_{L, K}\right),
$$

$$
\begin{aligned}
& E_{K L}^{(2)}=\frac{1}{2} U_{M, K} U_{M, L}, \\
& \mathbf{W}=\mathbf{W}^{(1)}, \quad W_{K}^{(1)}=-\phi_{, K}, \quad \mathbf{V}=\mathbf{V}^{(1)}, \quad V_{K}^{(1)}=\psi_{, K},
\end{aligned}
$$

and expansions

$$
\begin{aligned}
& I_{1}=I_{1}^{(1)}+I_{1}^{(2)}, \quad I_{1}^{(1)}=\mathbf{a} \cdot \mathbf{E}^{(1)} \cdot \mathbf{a}, \quad I_{1}^{(2)}=\mathbf{a} \cdot \mathbf{E}^{(2)} \cdot \mathbf{a}, \\
& I_{2}=I_{2}^{(1)}+I_{2}^{(2)}, \quad I_{2}^{(1)}=\operatorname{tr} \mathbf{E}^{(1)}, \quad I_{2}^{(2)}=\operatorname{tr} \mathbf{E}^{(2)}, \\
& I_{3}=I_{3}^{(1)}, \quad I_{3}^{(1)}=\mathbf{W}^{(1)} \cdot \mathbf{a}, \quad I_{4}=I_{4}^{(1)}, \quad I_{4}^{(1)}=\mathbf{V}^{(1)} \cdot \mathbf{a}, \\
& I_{1}=I I_{1}^{(2)}+\cdots, \quad I I_{1}^{(2)}=\mathbf{a} \cdot\left(\mathbf{E}^{(1)}\right)^{2} \cdot \mathbf{a}, \\
& I I_{2}^{(2)}=\operatorname{tr}\left(\mathbf{E}^{(1)}\right)^{2}, \quad I I_{3}^{(2)}=\mathbf{W}^{(1)} \cdot \mathbf{W}^{(1)}, \\
& I I_{4}^{(2)}=\mathbf{a} \cdot \mathbf{E}^{(1)} \cdot \mathbf{W}^{(1)}+\mathbf{W}^{(1)} \cdot \mathbf{E}^{(1)} \cdot \mathbf{a}, \quad I I_{5}^{(2)}=\mathbf{V}^{(1)} \cdot \mathbf{V}^{(1)}, \\
& I I_{6}^{(2)}=\mathbf{a} \cdot \mathbf{E}^{(1)} \cdot \mathbf{V}^{(1)}+\mathbf{V}^{(1)} \cdot \mathbf{E}^{(1)} \cdot \mathbf{a}, \quad I I_{7}^{(2)}=\mathbf{W}^{(1)} \cdot \mathbf{V}^{(1)}
\end{aligned}
$$

where a superscript enclosed in parentheses indicates the order of the quantity. We have written $\mathbf{W}$ and $\mathbf{V}$ as $\mathbf{W}^{(1)}$ and $\mathbf{V}^{(1)}$ to make formally superscripts of different terms homogeneous. Substituting (13) and (14) into (10)-(12), and keeping terms up to second order, we obtain the following second-order representations for $\mathbf{T}, \Pi$, and $\mathbf{h}$ :

$$
\begin{aligned}
& \mathbf{T}=\alpha_{1} \mathbf{a} \otimes \mathbf{a}+\alpha_{2} \mathbf{1}+\mathbf{T}^{(1)}+\mathbf{T}^{(2)}, \\
& \mathbf{\Pi}=-\beta \mathbf{a}+\mathbf{\Pi}^{(1)}+\mathbf{\Pi}^{(2)}, \\
& \mathbf{h}=\gamma \mathbf{a}+\mathbf{h}^{(1)}+\mathbf{h}^{(2)},
\end{aligned}
$$

where

$$
\begin{aligned}
& \mathbf{T}^{(1)}=\left(2 c_{1} I_{1}^{(1)}+c_{3} I_{2}^{(1)}+e_{1} I_{3}^{(1)}+f_{1} I_{4}^{(1)}\right) \mathbf{a} \otimes \mathbf{a}+\left(2 c_{2} I_{2}^{(1)}+c_{3} I_{1}^{(1)}+e_{2} I_{3}^{(1)}+f_{2} I_{4}^{(1)}\right) \mathbf{1}+c_{4}\left(\mathbf{a} \otimes \mathbf{E}^{(1)} \cdot \mathbf{a}+\mathbf{a} \cdot \mathbf{E}^{(1)} \otimes \mathbf{a}\right)+2 c_{5} \mathbf{E}^{(1)} \\
& +e_{3}\left(\mathbf{a} \otimes \mathbf{W}^{(1)}+\mathbf{W}^{(1)} \otimes \mathbf{a}\right)+f_{3}\left(\mathbf{a} \otimes \mathbf{V}^{(1)}+\mathbf{V}^{(1)} \otimes \mathbf{a}\right), \\
& \mathrm{T}^{(2)}=\left[2 c_{1} I_{1}^{(2)}+c_{3} I_{2}^{(2)}+3 \lambda_{1}\left(I_{1}^{(1)}\right)^{2}+2 \lambda_{3} I_{1}^{(1)} I_{2}^{(1)}+\lambda_{4}\left(I_{2}^{(1)}\right)^{2}+\lambda_{5} I I_{1}^{(2)}+\lambda_{7} I I_{2}^{(2)}+2 \nu_{1} I_{1}^{(1)} I_{3}^{(1)}+\nu_{2}\left(I_{3}^{(1)}\right)^{2}+\nu_{7} I I_{3}^{(2)}+\nu_{9} I I_{4}^{(2)}\right. \\
& \left.+\nu_{14} I_{2}^{(1)} I_{3}^{(1)}+2 \eta_{1} I_{1}^{(1)} I_{4}^{(1)}+\eta_{2}\left(I_{4}^{(1)}\right)^{2}+\eta_{7} I_{5}^{(2)}+\eta_{9} I I_{6}^{(2)}+\eta_{14} I_{2}^{(1)} I_{4}^{(1)}+\delta_{1} I_{3}^{(1)} I_{4}^{(1)}+\delta_{5} I_{7}^{(2)}\right] \mathrm{a} \otimes \mathbf{a}+\left[2 c_{2} I_{2}^{(2)}+c_{3} I_{1}^{(2)}\right. \\
& +3 \lambda_{2}\left(I_{2}^{(1)}\right)^{2}+\lambda_{3}\left(I_{1}^{(1)}\right)^{2}+2 \lambda_{4} I_{1}^{(1)} I_{2}^{(1)}+\lambda_{6} I I_{1}^{(2)}+\hat{\lambda}_{8} I I_{2}^{(2)}+2 \nu_{3} I_{2}^{(1)} I_{3}^{(1)}+\nu_{4}\left(I_{3}^{(1)}\right)^{2}+\nu_{8} I_{3}^{(2)}+\nu_{10} I_{4}^{(2)}+\nu_{14} I_{1}^{(1)} I_{3}^{(1)} \\
& \left.+2 \eta_{3} I_{2}^{(1)} I_{4}^{(1)}+\eta_{4}\left(I_{4}^{(1)}\right)^{2}+\eta_{8} I I_{5}^{(2)}+\eta_{10} I_{6}^{(2)}+\eta_{14} I_{1}^{(1)} I_{4}^{(1)}+\delta_{2} I_{3}^{(1)} I_{4}^{(1)}+\delta_{6} I I_{7}^{(2)}\right] \mathbf{1}+\left(\lambda_{5} I_{1}^{(1)}+\lambda_{6} I_{2}^{(1)}+\nu_{5} I_{3}^{(1)}+\eta_{5} I_{4}^{(1)}\right) \\
& \times\left(\mathbf{a} \otimes \mathbf{E}^{(1)} \cdot \mathbf{a}+\mathbf{a} \cdot \mathbf{E}^{(1)} \otimes \mathbf{a}\right)+c_{4}\left(\mathbf{a} \otimes \mathbf{E}^{(2)} \cdot \mathbf{a}+\mathbf{a} \cdot \mathbf{E}^{(2)} \otimes \mathbf{a}\right)+2\left(\lambda_{7} I_{1}^{(1)}+\lambda_{8} I_{2}^{(1)}+\nu_{6} I_{3}^{(1)}+\eta_{6} I_{4}^{(1)}\right) \mathbf{E}^{(1)}+2 c_{5} \mathbf{E}^{(2)} \\
& +\left(\nu_{9} I_{1}^{(1)}+\nu_{10} I_{2}^{(1)}+\nu_{11} I_{3}^{(1)}+\delta_{3} I_{4}^{(1)}\right)\left(\mathbf{a} \otimes \mathbf{W}^{(1)}+\mathbf{W}^{(1)} \otimes \mathbf{a}\right)+\left(\eta_{9} I_{1}^{(1)}+\eta_{10} I_{2}^{(1)}+\eta_{11} I_{4}^{(1)}+\delta_{4} I_{3}^{(1)}\right)\left(\mathbf{a} \otimes \mathbf{V}^{(1)}+\mathbf{V}^{(1)} \otimes \mathbf{a}\right) \\
& +3 \lambda_{9}\left(\mathbf{E}^{(1)}\right)^{2}+\nu_{12} \mathbf{W}^{(1)} \otimes \mathbf{W}^{(1)}+\eta_{12} \mathbf{V}^{(1)} \otimes \mathbf{V}^{(1)}+\nu_{13}\left(\mathbf{a} \otimes \mathbf{E}^{(1)} \cdot \mathbf{W}^{(1)}+\mathbf{W}^{(1)} \cdot \mathbf{E}^{(1)} \otimes \mathbf{a}+\mathbf{W}^{(1)} \otimes \mathbf{E}^{(1)} \cdot \mathbf{a}+\mathbf{a} \cdot \mathbf{E}^{(1)} \otimes \mathbf{W}^{(1)}\right) \\
& +\eta_{13}\left(\mathbf{a} \otimes \mathbf{E}^{(1)} \cdot \mathbf{V}^{(1)}+\mathbf{V}^{(1)} \cdot \mathbf{E}^{(1)} \otimes \mathbf{a}+\mathbf{V}^{(1)} \otimes \mathbf{E}^{(1)} \cdot \mathbf{a}+\mathbf{a} \cdot \mathbf{E}^{(1)} \otimes \mathbf{V}^{(1)}\right)+\delta_{7}\left(\mathbf{W}^{(1)} \otimes \mathbf{V}^{(1)}+\mathbf{V}^{(1)} \otimes \mathbf{W}^{(1)}\right), \\
& \Pi^{(1)}=-\left(2 \epsilon_{1} I_{3}^{(1)}+e_{1} I_{1}^{(1)}+e_{2} I_{2}^{(1)}+g_{1} I_{4}^{(1)}\right) \mathbf{a}-2 e_{3} \mathbf{E}^{(1)} \cdot \mathbf{a}-2 \epsilon_{2} \mathbf{W}^{(1)}-g_{2} \mathbf{V}^{(1)}, \\
& \Pi^{(2)}=-\left[e_{1} I_{1}^{(2)}+e_{2} I_{2}^{(2)}+3 \mu_{1}\left(I_{3}^{(1)}\right)^{2}+\mu_{2} I I_{3}^{(2)}+\nu_{1}\left(I_{1}^{(1)}\right)^{2}+2 \nu_{2} I_{3}^{(1)} I_{1}^{(1)}+\nu_{3}\left(I_{2}^{(1)}\right)^{2}+2 \nu_{4} I_{3}^{(1)} I_{2}^{(1)}+\nu_{5} I I_{1}^{(2)}+\nu_{6} I I_{2}^{(2)}\right. \\
& \left.+\nu_{11} I_{4}^{(2)}+\nu_{14} I_{1}^{(1)} I_{2}^{(1)}+2 \zeta_{1} I_{3}^{(1)} I_{4}^{(1)}+\zeta_{2}\left(I_{4}^{(1)}\right)^{2}+\zeta_{4} I I_{5}^{(2)}+\zeta_{5} I I_{7}^{(2)}+\delta_{1} I_{1}^{(1)} I_{4}^{(1)}+\delta_{2} I_{2}^{(1)} I_{4}^{(1)}+\delta_{4} I I_{6}^{(2)}\right] \mathrm{a}
\end{aligned}
$$




$$
\begin{aligned}
& -2\left(\nu_{9} I_{1}^{(1)}+\nu_{10} I_{2}^{(1)}+\nu_{11} I_{3}^{(1)}+\delta_{3} I_{4}^{(1)}\right) \mathbf{E}^{(1)} \cdot \mathbf{a}-2 e_{3} \mathbf{E}^{(2)} \cdot \mathbf{a}-2\left(\mu_{2} I_{3}^{(1)}+\nu_{7} I_{1}^{(1)}+\nu_{8} I_{2}^{(1)}+\zeta_{3} I_{4}^{(1)}\right) \mathbf{W}^{(1)} \\
& -\left(\zeta_{5} I_{3}^{(1)}+\zeta_{6} I_{4}^{(1)}+\delta_{5} I_{1}^{(1)}+\delta_{6} I_{2}^{(1)}\right) \mathbf{V}^{(1)}-2 \nu_{13}\left(\mathbf{E}^{(1)}\right)^{2} \cdot \mathbf{a}-2 \nu_{12} \mathbf{E}^{(1)} \cdot \mathbf{W}^{(1)}-2 \delta_{7} \mathbf{E}^{(1)} \cdot \mathbf{V}^{(1)} \\
\mathbf{h}^{(1)}= & \left(2 \kappa_{1} I_{4}^{(1)}+f_{1} I_{1}^{(1)}+f_{2} I_{2}^{(1)}+g_{1} I_{3}^{(1)}\right) \mathbf{a}+2 f_{3} \mathbf{E}^{(1)} \cdot \mathbf{a}+g_{2} \mathbf{W}^{(1)}+2 \kappa_{2} \mathbf{V}^{(1)} \\
\mathbf{h}^{(2)}= & {\left[f_{1} I_{1}^{(2)}+f_{2} I_{2}^{(2)}+3 \xi_{1}\left(I_{4}^{(1)}\right)^{2}+\xi_{2} I I_{5}^{(2)}+\eta_{1}\left(I_{1}^{(1)}\right)^{2}+2 \eta_{2} I_{4}^{(1)} I_{1}^{(1)}+\eta_{3}\left(I_{2}^{(1)}\right)^{2}+2 \eta_{4} I_{4}^{(1)} I_{2}^{(1)}+\eta_{5} I I_{1}^{(2)}+\eta_{6} I_{2}^{(2)}+\eta_{11} I I_{6}^{(2)}\right.} \\
& \left.+\eta_{14} I_{1}^{(1)} I_{2}^{(1)}+2 \zeta_{2} I_{3}^{(1)} I_{4}^{(1)}+\zeta_{1}\left(I_{3}^{(1)}\right)^{2}+\zeta_{3} I_{3}^{(2)}+\zeta_{6} I I_{7}^{(2)}+\delta_{1} I_{1}^{(1)} I_{3}^{(1)}+\delta_{2} I_{2}^{(1)} I_{3}^{(1)}+\delta_{3} I I_{4}^{(2)}\right] \mathbf{a} \\
& +2\left(\eta_{9} I_{1}^{(1)}+\eta_{10} I_{2}^{(1)}+\eta_{11} I_{4}^{(1)}+\delta_{4} I_{3}^{(1)}\right) \mathbf{E}^{(1)} \cdot \mathbf{a}+2 f_{3} \mathbf{E}^{(2)} \cdot \mathbf{a}+\left(\zeta_{5} I_{3}^{(1)}+\zeta_{6} I_{4}^{(1)}+\delta_{5} I_{1}^{(1)}+\delta_{6} I_{2}^{(1)}\right) \mathbf{W}^{(1)} \\
& +2\left(\xi_{2} I_{4}^{(1)}+\eta_{7} I_{1}^{(1)}+\eta_{8} I_{2}^{(1)}+\zeta_{4} I_{3}^{(1)}\right) \mathbf{V}^{(1)}+2 \eta_{13}\left(\mathbf{E}^{(1)}\right)^{2} \cdot \mathbf{a}+2 \delta_{7} \mathbf{E}^{(1)} \cdot \mathbf{W}^{(1)}+2 \eta_{12} \mathbf{E}^{(1)} \cdot \mathbf{V}^{(1)}
\end{aligned}
$$

\section{LINEAR CONSTITUTIVE RELATIONS}

The linear or first-order constitutive relations (16), (18), and (20), when terms are rearranged according to the order of dependence on $\mathbf{E}^{(1)}, \mathbf{W}^{(1)}$, and $\mathbf{V}^{(1)}$, are

$$
\begin{aligned}
\mathbf{T}^{(1)}= & \left(2 c_{1} I_{1}^{(1)}+c_{3} I_{2}^{(1)}\right) \mathbf{a} \otimes \mathbf{a}+\left(2 c_{2} I_{2}^{(1)}+c_{3} I_{1}^{(1)}\right) \mathbf{1}+c_{4}\left(\mathbf{a} \otimes \mathbf{E}^{(1)} \cdot \mathbf{a}+\mathbf{a} \cdot \mathbf{E}^{(1)} \otimes \mathbf{a}\right)+2 c_{5} \mathbf{E}^{(1)}+e_{1} I_{3}^{(1)} \mathbf{a} \otimes \mathbf{a}+e_{2} I_{3}^{(1)} \mathbf{1} \\
& +e_{3}\left(\mathbf{a} \otimes \mathbf{W}^{(1)}+\mathbf{W}^{(1)} \otimes \mathbf{a}\right)+f_{1} I_{4}^{(1)} \mathbf{a} \otimes \mathbf{a}+f_{2} I_{4}^{(1)} \mathbf{1}+f_{3}\left(\mathbf{a} \otimes \mathbf{V}^{(1)}+\mathbf{V}^{(1)} \otimes \mathbf{a}\right), \\
\Pi^{(1)}= & -\left(e_{1} I_{1}^{(1)}+e_{2} I_{2}^{(1)}\right) \mathbf{a}-2 e_{3} \mathbf{E}^{(1)} \cdot \mathbf{a}-2 \epsilon_{1} I_{3}^{(1)} \mathbf{a}-2 \epsilon_{2} \mathbf{W}^{(1)}-g_{1} I_{4}^{(1)} \mathbf{a}-g_{2} \mathbf{V}^{(1)}, \\
\mathbf{h}^{(1)}= & \left(f_{1} I_{1}^{(1)}+f_{2} I_{2}^{(1)}\right) \mathbf{a}+2 f_{3} \mathbf{E}^{(1)} \cdot \mathbf{a}+g_{1} I_{3}^{(1)} \mathbf{a}+g_{2} \mathbf{W}^{(1)}+2 \kappa_{1} I_{4}^{(1)} \mathbf{a}+2 \kappa_{2} \mathbf{V}^{(1)} .
\end{aligned}
$$

These can be written a matrix form. ${ }^{89}$

\section{SMALL DEFORMATIONS AND STRONG ELECTRIC FIELDS}

In this case, (15), (16), (18), and (20) remain the same. Equations (17), (19), and (21) reduce to

$$
\begin{aligned}
& \mathbf{T}^{(2)}=\left[\nu_{2}\left(I_{3}^{(1)}\right)^{2}+\nu_{7} I I_{3}^{(2)}\right] \mathbf{a} \otimes \mathbf{a}+\left[\nu_{4}\left(I_{3}^{(1)}\right)^{2}+\nu_{8} I I_{3}^{(2)}\right] \mathbf{1}+\nu_{11} I_{3}^{(1)}\left(\mathbf{a} \otimes \mathbf{W}^{(1)}+\mathbf{W}^{(1)} \otimes \mathbf{a}\right)+\nu_{12} \mathbf{W}^{(1)} \otimes \mathbf{W}^{(1)}, \\
& \Pi^{(2)}=-\left[3 \mu_{1}\left(I_{3}^{(1)}\right)^{2}+\mu_{2} I I_{3}^{(2)}\right] \mathbf{a}-2 \mu_{2} I_{3}^{(1)} \mathbf{W}^{(1)}, \\
& \mathbf{h}^{(2)}=\left[\zeta_{1}\left(I_{3}^{(1)}\right)^{2}+\zeta_{3} I I_{3}^{(2)}\right] \mathbf{a}+\zeta_{5} I_{3}^{(1)} \mathbf{W}^{(1)},
\end{aligned}
$$

which can also be written in a matrix form. ${ }^{8,9}$

\section{CONCLUSIONS}

Constitutive relations (22) imply that for a linear theory, contributions to stresses, electric polarization, and the equilibrated stresses from the mechanical strains, the electric field, and the porosity gradients are additive. However, such is not the case in the nonlinear theory as should be evident from the second-order constitutive relations (15) $-(21)$. When the changes in porosity are also infinitesimal, then the abovestated constitutive relations can be suitably modified. In them, the material parameters will depend upon the initial value of the porosity.

\section{ACKNOWLEDGMENTS}

This work was supported by the U.S. Army Research Office Grant No. DAAH 04-93-G-0214 to the University of Missouri-Rolla, and a matching grant from the Missouri Research and Training Center. The Virginia Polytechnic Institute and State University acted as a subcontractor to the University of Missouri-Rolla.
1 D. F. Nelson, Electric, Optic, and Acoustic Interactions in Dielectrics (Wiley, New York, 1979), pp. 490-513.

${ }^{2}$ M. Ciarletta and A. Scalia, "Thermodynamic theory for porous piezoelectric materials," Meccanica 28 (4), 303-308 (1993).

${ }^{3} \mathrm{M}$. Ciarletta and A. Scalia, "Minimum principle in the linear theory of porous piezoelectric materials," Rend. Circolo Mat. Palermo, Ser. II 42, 65-81 (1993).

${ }^{4} \mathrm{M}$. Ciarletta and A. Scalia, "On thermopiezoelectricity for porous materials," J. Thermal Stresses 16, 329-349 (1993).

${ }^{5} \mathrm{~J}$. W. Nunziato and S. C. Cowin, "A nonlinear theory of elastic materials with voids," Arch. Rat. Mech. Anal. 72, 175-201 (1979).

${ }^{6}$ S. L. Passman and R. C. Batra, "A thermomechanical theory for a porous anisotropic elastic solid with inclusions," Arch. Rat. Mech. Anal. 72, 175201 (1979).

${ }^{7}$ Q.-S. Zheng, "On Transversely Isotropic, Orthotropic and Relatively Isotropic Functions of Symmetric Tensors, Skew-symmetric Tensors, and Vectors," Int. J. Eng. Sci. 31 (10), 1399-1453 (1993).

${ }^{8}$ J. S. Yang and R. C. Batra, "A second-order theory for piezoelectric materials," J. Acoust. Soc. Am. 97, 280-288 (1995).

${ }^{9}$ R. C. Batra and J. S. Young, "Second-order constitutive relations for transversely isotropic piezoelectric porous materials" (available from the authors). 